МРНТИ 29.27.31; 29.27.47

\author{
Исмагамбетова Т.Н., Габдуллин М.Т. ${ }^{1,2}$ \\ ${ }^{1}$ НИИЭТФ, Казахский национальный университет им. аль-Фараби, \\ Казахстан, г. Алматы \\ ${ }^{2}$ Казахстанско-Британский технический университет, \\ Казахстан, г. Алматы, e-mail: gabdullin@physics.kz

\section{ПАРНЫЕ КОРРЕАЯЦИОННЫЕ ФУНКЦИИ ПАОТНОЙ ВОАОРОАНОЙ ПААЗМЫ}

Были исследованы структурные свойства плотной неидеальной водородной плазмы. Потенциалы взаимодействия частиц, учитывающие квантово-механические эффекть дифракции и симметрии, были использованы в качестве модели взаимодействия. Электронэлектронные парные корреляционные функции были получены при решении интегрального уравнения Орнштейна-Цернике в гипперцепном приближении на основе потенциалов взаимодействия. Квантово-механический эффект симметрии рассматривался А^я разных направлений спинов электронов. Эффект симметрии учитывает принцип исключения Паули. Влияние эффекта симметрии как квантово-механического эффекта более выражено на малых расстояниях и в более плотной плазме. Антипаралмельное направление электронных спинов увеличивает вероятность нахождения электронов на расстоянии $\mathrm{R}$ Аруг от Аруга, парамлельное направление уменьшает эту вероятность из-за запрета на присутствие Авух электронов с одинаковыми спинами в одном и том же состоянии. Результаты данной работы были сравнены с результатами метода классического сопоставления ("classical mapping"). Основная идея классического сопоставления состоит в том, чтобы установить взаимосвязь между квантовой и эталонной классической системами, такую, чтобы структура классической системы была такая же, как у рассматриваемой квантовой системы. Результаты Аанной работы показывают хорошее согласие с результатами метода классического сопоставления в гипперцепном приближении с мостиковой функцией и компьютерного модемирования.

Кмючевые слова: неидеальная плазма, парные корреляционные функции.

\author{
Ismagambetova T.N. ${ }^{1}$, Gabdullin M.T. ${ }^{1,2}$ \\ 'IETP, al-Farabi Kazakh National University, Kazakhstan, Almaty \\ ${ }^{2}$ Kazakh-British Technical University, Kazakhstan, Almaty, e-mail: gabdullin@physics.kz
}

\title{
Pair correlation functions of a dense hydrogen plasma
}

The structural properties of a dense non-ideal hydrogen plasma were investigated. The interaction potentials of particles, taking into account the quantum-mechanical effects of diffraction and symmetry, were used as an interaction model. The electron-electron pair correlation functions were obtained by solving the Ornstein-Zernike integral equation in the hypernetted-chain approximation based on the interaction potentials. The quantum mechanical symmetry effect was considered for different directions of electron spins. The symmetry effect takes into account the Pauli exclusion principle. The effect of the symmetry effect as a quantum mechanical effect is more pronounced at small distances and in a denser plasma. The antiparallel direction of electron spins increases the probability of finding electrons at a distance $\mathrm{R}$ from each other, the parallel direction reduces this probability due to the prohibition of the presence of two electrons with identical spins in the same state. The results of this work were compared with the results of the classical comparison method ("classical mapping"). The main idea of the classical comparison is to establish the relationship between the quantum and reference classical systems, such that the structure of the classical system is the same as that of the quantum system in question. The results of this paper show good agreement with the results of the classical mapping in the hypernettedchain approximation with the bridge function and computer simulation.

Key words: non-ideal plasma, pair correlation functions. 


\author{
Исмагамбетова Т.Н. ${ }^{1}$, Габдуммин М.Т. ${ }^{1,2}$ \\ 1ЭТФҒЗИ, әл-Фараби атындағы Қазақ ұлттық университеті, \\ Қазақстан, Алматы қ. \\ ${ }^{2}$ Қазақстан-Британ техникалық университеті, \\ Қазақстан, Алматы қ., е-mail: gabdullin@physics.kz
}

\title{
Тығыз сутекті плазманың жұп корреляциямық функциялары
}

\begin{abstract}
Тығыз идеал емес сутекті плазманың құрылымдық қасиеттері зерттелді. Аифракция мен симметрияның кванттық-механикалық эффектілерін ескеретін бөлшектердің әсерлесу потенциалдары әсерлесу үлгісі ретінде қолданылды. Электрон-электрон жұп корреляциялық, функциялары әсерлесу потенциалдарының негізінде гипперцепты жуықтаудағы ОрнштейнЦернике интегра^ды теңдеуін шешу барысында алынды. Симметрияның кванттық-механикалық, эффектісі электрон спиндарының әртүрлі бағыттары үшін қарастырылды. Симметрия эффектісі Паулидің шығарып тастау принципін ескереді. Симметрия эффектісінің кванттық-механикалық, эффект ретіндегі әсері жауын қашықтықтарда және тығыз плазмада жақсы көрінеАі. Электрон спиндарының паралмель емес бағыттары электрондардың бір-бірінен $R$ қашықтықта болу мүмкіндігін үлкейтеді, ал паралмель бағыт бұл мүмкіндікті кішірейтеді, себебі бір күйде спиндары бірдей екі электрон болуына тыйым салынған. Берілген жұмыстың нәтижелері классикалық, салыстыру әдісінің (“classical mapping”) нәтижелерімен салыстырылған. К^ассикалық салыстырудың негізгі ойы - кванттық, және эталондық, классикалық жүйелердің арасында классикалық, жүйенің құрылымы қарастырылып отырған кванттық жүйенікімен бірдей болатындай байланыс орнату. Берілген жұмыстың нәтижелері көпірлік функциясы бар гипперцепты жуықтаудағы классикалық салыстыру әдісі мен компьютерлік модельдеу нәтижелерімен жақсы ұйқастық көрсетеАі.
\end{abstract}

Түйін сөздер: идел емес плазма, жұптық корреляциялық функция.

\section{1. Введение}

В данной работе рассматривается плотная водородная плазма, представляющая значительный интерес как в теоретическом, так и в практическом плане, например, неидеальная плазма, встречающаяся в термоядерных реакторах. В плотной или неидеальной плазме значительную роль играют межчастичные взаимодействия. В такой плазме среднее межчастичное расстояние имеет один порядок $\mathrm{c}$ тепловой длиной волной частиц. В плотной плазме высока вероятность столкновении с таким максимальным сближением, что становится важным учет волновой природы сталкивающихся частиц.

Потенциалы взаимодействия [1-3], используемые в этой статье, учитывают квантовомеханические эффекты дифракции и симметрии на малых расстояниях:

$$
u_{a b}(r)=\frac{e_{a} e_{b}}{r}\left(1-t h\left(\sqrt{2} \frac{\lambda_{a b}^{2}}{a_{B}^{2}+b r^{2}}\right) e^{-t h\left(\sqrt{2} \frac{\lambda_{a b}^{2}}{a_{B}^{2}+b r^{2}}\right)}\right)\left(1-e^{-\frac{r}{\lambda_{a b}}}\right)-\delta_{a e} \delta_{b e} k_{B} T \ln \left(1-\frac{1}{2} e^{-\frac{r^{2}}{\lambda_{e e}^{2}}}\right)
$$

где $\boldsymbol{e}_{\boldsymbol{a}} \boldsymbol{e}_{\boldsymbol{b}}$ - заряд частиц а и $\mathrm{b}, \lambda_{a b}=\frac{\hbar}{\sqrt{2 \pi m_{a b} k_{B} T}}$

$$
\begin{gathered}
\text { - тепловая длина волны де Бройля, } \\
m_{a b}=\frac{m_{a} m_{b}}{\left(m_{a}+m_{b}\right)}-\text { приведенная масса, } \\
r_{D}=\left(\frac{k_{B}}{4 \pi e^{2} \sum_{j} n_{j} Z_{j}^{2}}\right)^{1 / 2}-\text { радиус Дебая, также }
\end{gathered}
$$

безразмерные параметры: $\Gamma=\frac{(\mathrm{Ze})^{2}}{a k_{B} T}-$ параметр связи, $\quad r_{s}=\frac{a}{a_{B}} \quad-$ параметр плотности, $a=\left(\frac{3}{4 \pi n_{e}}\right)^{1 / 3}-$ среднее расстояние между электронами, $a_{B}-$ радиус Бора. Первое слагаемое учитывает эффект дифракции, второе - 
эффект симметрии (усредненное направление электронных спинов).

Параметр вырождения для электронной компоненты определяется следующим выражением:

$$
\Theta=\frac{k_{B} T}{E_{F}}=2\left(\frac{4}{9 \pi}\right)^{\frac{2}{3}} Z^{\frac{5}{3}} \frac{r_{s}}{\Gamma},
$$

Где $E_{F}$ - энергия Ферми электронов, $\theta>>1$ - классическая плазма, $\theta \geq 1$ - слабо вырожденная плазма, $\theta<1$ - вырожденная плазма.

Для учета разных направлений спина электрона использовалась следующая формула вместо второго слагаемого в формуле (1):

$$
U_{e c, 0}^{s(\mathrm{)})}(r)=-k_{B} T \ln \left(1 \pm \exp \left(-\frac{r^{2}}{\lambda_{c e}^{2}}\right)\right),
$$

где $\mathrm{S}=1$ соответствует параллельным спинам, $\mathrm{S}=0$ - антипараллельным спинам.

\section{2. Парные корреляционные функции}

Парные корреляционные функции g(r) были рассчитаны на основе интегрального уравнения Орнштейна-Цернике [4]:

$$
h(\vec{r})=C(\vec{r})+n \int C\left(\overrightarrow{r_{3}}-\overrightarrow{r_{1}}\right) h\left(\left|\overrightarrow{r_{3}}-\overrightarrow{r_{2}}\right|\right) d r_{3}
$$

в гипперцепном приближении (HNC):

$$
C_{H N C}(r)=h(r)-\ln g(r)-\frac{u(r)}{k_{B} T},
$$

где $\overrightarrow{h(\vec{r})}=g(\vec{r})-1 \quad-$ полная корреляционная функция, $C(r)$ - прямая корреляционная функция, $u(r)-$ потенциал взаимодействия. Уравнения (4) и (5) были рассчитаны численными методами.

На рисунке 1 представлены электрон-электронные потенциалы взаимодействия, полученные в данной работе, в сравнении с [5]. На рисунке 1 показаны графики потенциалов взаимодействия при различных значениях параметра вырождения $\mathrm{t}=\theta=0$ (черная), 0.1 (красные квадратики), 1 (синие кружочки), 10 (сиреневые квадратики). Синяя $(\mathrm{t}=\theta=1)$ и бирюзовая $(\mathrm{t}=\theta=10)$ линии - результаты на основе потенциала (1) при усредненном направлении электронных спинов.

На рисунках 2-4 представлены графики электрон-электронных парных корреляционных функций, рассчитанных на основе потенциала (1), в сравнении с работой [6]. На рисунке 2 представлены электрон-электронные парные корреляционные функции при $\mathrm{r}_{\mathrm{s}}=1, \quad \Gamma=0.5$. Сплошные линии - результаты классического сопоставления (“classical mapping”) в гипперцепном приближении с мостиковой функцией [710], полученной в [11] на основе [12-15] (HNCB), точечные линии - результаты квантового моделирования Монте-Карло (QMC). Черные линии - для параллельных спинов, красные линии - для антипараллельных спинов. На рисунке 3 показаны электрон-электронные парные корреляционные функции при $\mathrm{r}_{\mathrm{s}}=1$, $\Gamma=0.5$ и $\mathrm{r}_{\mathrm{s}}=6, \Gamma=3$. Сплошные линии $-\mathrm{HNCB}$, пунктирные линии - HNC [16], точечные линии - QMC [17]. Черные линии $-\mathrm{r}_{\mathrm{s}}=1$, красные линии $-\mathrm{r}_{\mathrm{s}}=6$. Бирюзовая и сиреневая линии - на основе потенциала (1) при $\mathrm{r}_{\mathrm{s}}=1$ и $\mathrm{r}_{\mathrm{s}}=6$. На рисунке 4 показаны электрон-электронные парные корреляционные функции при $\mathrm{r}_{\mathrm{s}}=1, \Gamma=0.07$ и $\mathrm{r}_{\mathrm{s}}=6$, $\Gamma=0.4$. Сплошные линии $-\mathrm{HNCB}$, пунктирные линии - HNC, точечные линии - QMC. Черные линии $-r_{s}=1$, красные линии $-r_{s}=6$. Зеленая и синяя линии - на основе потенциала (1) при $\mathrm{r}_{\mathrm{s}}=1$ и $\mathrm{r}_{\mathrm{s}}=6$.

На рисунках 5-6 представлены электронэлектронные парные корреляционные функции, полученные в данной работе, в сравнении с [18].

На рисунке 5 представлены электрон-электронные парные корреляционные функции при $\mathrm{r}_{\mathrm{s}}=6, \mathrm{t}=\theta=0.5$ (черная линия), 1 (красная линия), 4 (зеленая линия), 8 (сиреневая линия). Сплошными линиями обозначены результаты классического сопоставления в гипперцепном приближении с мостиковой функцией, квадратиками компьютерного моделирования Монте-Карло (PIMC). На рисунке 6 показаны электрон-электронные парные корреляционные функции при $\mathrm{r}_{\mathrm{s}}=6, \mathrm{t}=\theta=0.5$ (черная линия), 1 (красная линия), 4 (зеленая линия), 8 (сиреневая линия). Сплошными линиями обозначены результаты классического сопоставления в гипперцепном приближении с мостиковой функцией, кружочками классического сопоставления (PDW). Сплошная синяя и бирюзовая линии обозначают электрон- 
электронные парные корреляционные функции на основе потенциала (1) при $\mathrm{t}=\theta=4$ и 8.

Эффект симметрии учитывает принцип Паули, который запрещает одновременное присутствие двух одинаковых частиц с полуцелым спином (в данном случае электронов) в одном квантовом состоянии, что уменьшает вероятность нахождения частиц на расстоянии $\mathrm{r}$ друг от друга. Влияние эффекта симметрии как квантово-механического эффекта более выражено на малых расстояниях и в более плотной плазме.

Результаты данной работы показывают хорошее согласие с результатами метода классического сопоставления ("classical mapping") в гипперцепном приближении с мостиковой функцией и компьютерного моделирования.
Основная идея классического сопоставления состоит в том, чтобы установить взаимосвязь между квантовой и эталонной классической системами, такую, чтобы структура классической системы была такая же, как у рассматриваемой квантовой системы. С помощью потенциала взаимодействия, учитывающего квантовые эффекты, мы можем решить корреляционные функции эталонной классической системы, используя классические методы многих тел, а именно теории жидких состояний. Этот метод, предложенный Дхарма-Варданой и др. [19], классический метод сопоставления, был успешно применен к различным электронным системам с небольшими вычислительными затратами [20-25].

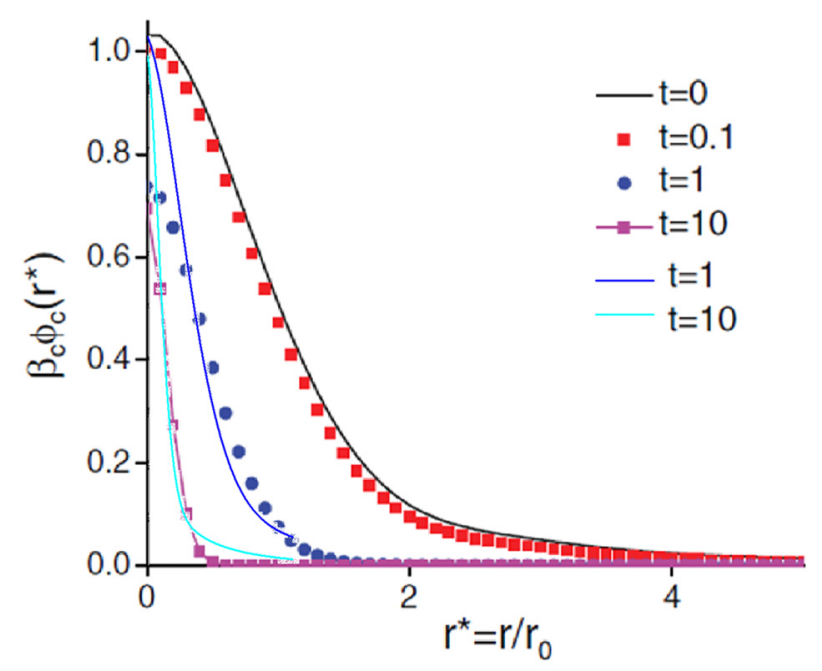

Рисунок 1 - Парный потенциал Паули для $\mathrm{t}=\theta=0,0.1,1,10$

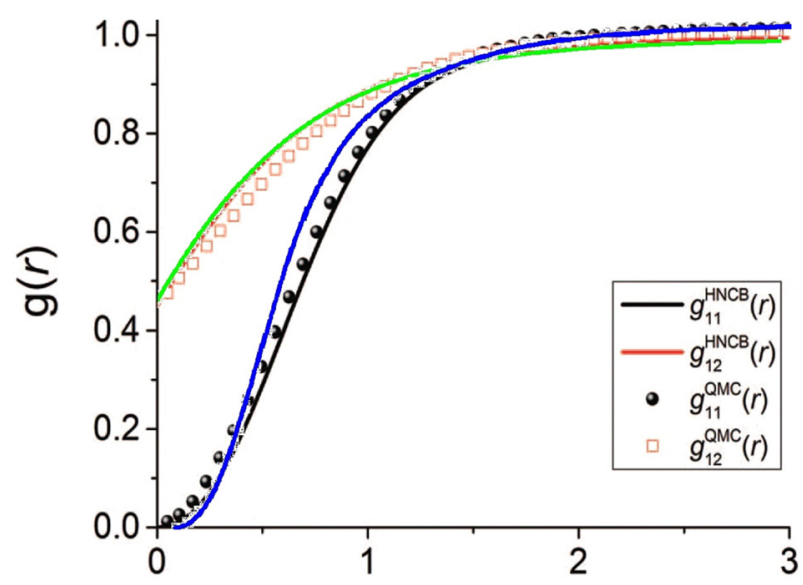

Рисунок 2 - Электрон-электронные парные корреляционные функции при $\mathrm{r}_{\mathrm{s}}=1, \Gamma=0.5$ 


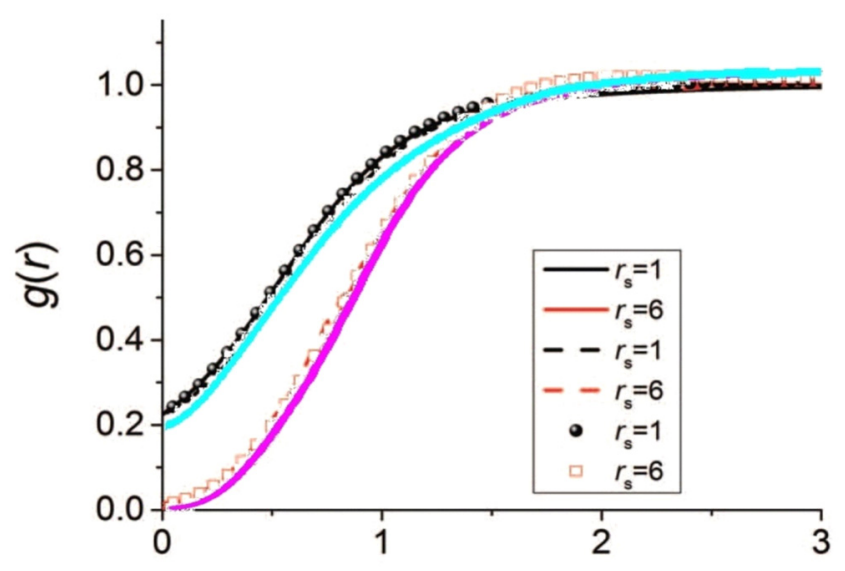

Рисунок 3 - Электрон-электронные парные корреляционные функции при $\mathrm{r}_{\mathrm{s}}=1, \Gamma=0.5$ и $\mathrm{rs}=6, \Gamma=3$

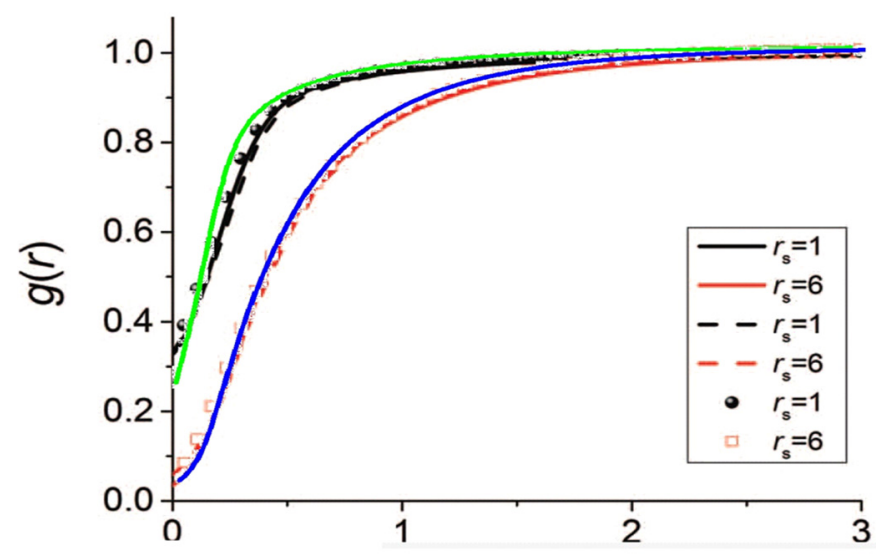

Рисунок 4 - Электрон-электронные парные корреляционные функции при $\mathrm{r}_{\mathrm{s}}=1, \Gamma=0.07$ и $\mathrm{rs}=6, \Gamma=0.4$

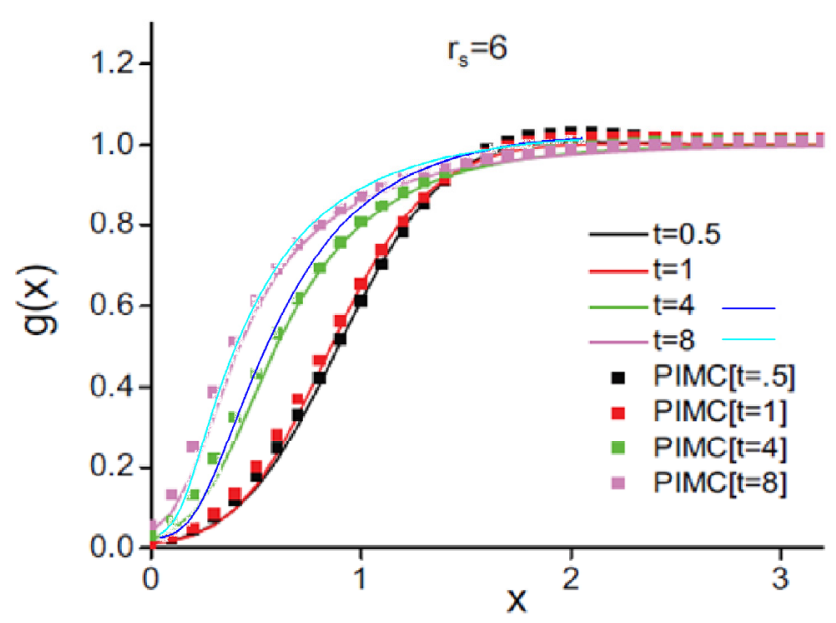

Рисунок 5 - Электрон-электронные парные корреляционные функции при $\mathrm{r}_{\mathrm{s}}=6, \mathrm{t}=\theta=0.5,1,4,8$ 


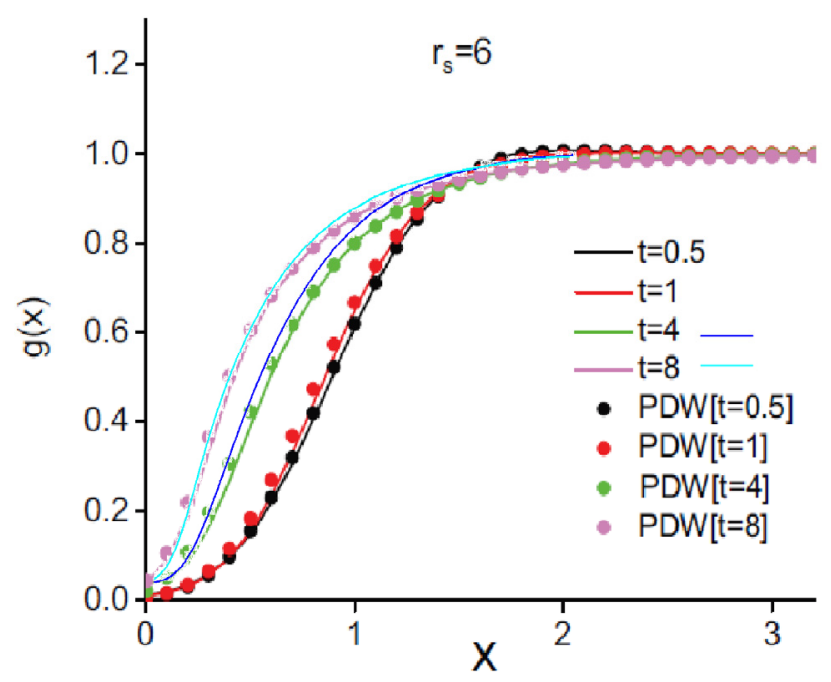

Рисунок 6 - Электрон-электронные парные корреляционные функции при $\mathrm{r}_{\mathrm{s}}=6, \mathrm{t}=0=0.5,1,4,8$

\section{3. Выводы}

Эффект симметрии учитывает принцип исключения Паули. Влияние эффекта симметрии как квантово-механического эффекта более выражено на малых расстояниях и в более плотной плазме. Антипараллельное направление электронных спинов увеличивает вероятность нахождения электронов на расстоянии $\mathrm{R}$ друг от друга, параллельное направление уменьшает эту вероятность из-за запрета на присутствие двух электронов с одинаковыми спинами в одном и том же состоянии.

Эта работа была поддержана Министерством образования и науки Казахстана по гранту на проект №AP05134366.

\section{Литература}

1 Moldabekov Zh.A., Ramazanov T.S., Dzhumagulova K.N. Pair Interaction Potential of Particles for Two-Component Plasma // Contrib.Plasma Phys. - 2012. - Vol. 52. - P. 207-210.

2 Ramazanov T.S., Moldabekov Zh.A., Gabdullin M.T., and Ismagambetova T.N. Interaction potentials and thermodynamic properties of two component semiclassical plasma // Phys. Plasmas. - 2014. - Vol. 21. - P. 012706.

3 Gabdullin M.T., Ramazanov T.S., Muratov M.M., Ismagambetova T.N., Akhtanova G.B., and Goree J.A. Structural Characteristics and Equation of State of the Complex Plasmas // Contrib. Plasma Phys. - 2015. - Vol. 55 (5). - P. 366-372.

4 Goodstein D.L. States of Matter. - Dover publications, Inc., 2002. - 512 p.

5 Dutta S., and Dufty J. Classical representation of a quantum system at equilibrium: Theory // Phys. Rev. E. - 2013. - Vol. 87. - P. 032101.

6 Liu Y., and Wu J. A bridge-functional-based classical mapping method for predicting the correlation functions of uniform electron gases at finite temperature // The Journal of Chemical Physics. - 2014. - Vol. 140. - P. 084103.

7 Du Q. H., Beglov D., and Roux B. Solvation Free Energy of Polar and Nonpolar Molecules in Water: An Extended Interaction Site Integral Equation Theory in Three Dimensions // J. Phys. Chem. B. - 2000. - Vol. 104. - P. 796-805.

8 Ballone P., Pastore G., Galli G., and Gazzillo D. Additive and non-additive hard sphere mixtures // Mol. Phys. - 1986. Vol. 59. - P. 275-290.

9 Duh D. M. and Henderson D. Integral equation theory for Lennard-Jones fluids: The bridge function and applications to pure fluids and mixtures // J. Chem. Phys.- 1996. - Vol. 104. - P. 6742.

10 Kovalenko A. and Hirata F. Hydration free energy of hydrophobic solutes studied by a reference interaction site model with a repulsive bridge correction and a thermodynamic perturbation method // J. Chem. Phys.- 2000. - Vol. 113. - P. 2793.

11 Zhao S., Feng P., and Wu J. A liquid-state theory for electron correlation functions and thermodynamics // Chem. Phys. Lett. - 2013. - Vol. 556. - P. 336-340.

12 Rosenfeld Y. Free-Energy Model for the Inhomogeneous Hard-Sphere Fluid Mixture and Density-Functional Theory of Freezing // Phys. Rev. Lett. - 1989. - Vol. 63. - P. 980.

13 Kierlik E. and Rosinberg M. L. Density-functional theory for inhomogeneous fluids: Adsorption of binary mixtures // Phys. Rev. A. - 1991. - Vol. 44. - P. 5025. 324

14 Lowen H. Melting, freezing and colloidal suspensions // Phys. Rep.-Rev. Sec. Phys. Lett. - 1994. - Vol. 237. - P. 249- 
$15 \mathrm{Yu}$ Y.X. and Wu J.Z. Structures of hard-sphere fluids from a modified fundamental-measure theory // J. Chem. Phys. 2002. - Vol. 117. - P. 10156.

16 Dutta S. and Dufty J. Uniform electron gas at warm, dense matter conditions // Europhys. Lett. - 2013. - Vol. 102. - P. 67005.

17 Brown E.W., Clark B.K., DuBois J.L., and Ceperley D.M. Path-Integral Monte Carlo Simulation of the Warm Dense Homogeneous Electron Gas // Phys. Rev. Lett. - 2013. - Vol. 110. - P. 146405.

18 Dutta S., and Dufty J. Classical representation of a quantum system at equilibrium: Applications // Phys. Rev. E. - 2013. Vol. 87. - P. 032102.

19 Dharma-Wardana M. W. C., and Perrot F. A simple classical mapping of the spin-polarized quantum electron gas: distribution functions and local field corrections // Phys. Rev. Lett. - 2000. - Vol. 84. - P. 959.

20 Dharma-Wardana M.W.C. Spin and temperature dependent study of exchange and correlation in thick two-dimensional electron layers // Phys. Rev. B. - 2005. - Vol. 72. - P. 125339.

21 Dharma-Wardana M.W.C., Perrot F. Structure of the local-field factor of the 2D electron fluid. Possible evidence for correlated scattering of electron pairs // Europhys. Lett. - 2003. - Vol. 63. - P. 660.

22 Dharma-Wardana M.W.C., Murillo M.S. Pair-distribution functions of two-temperature two-mass systems: Comparison of molecular dynamics, classical-map hypernetted chain, quantum Monte Carlo, and Kohn-Sham calculations for dense hydrogen // Phys. Rev. E. - 2008. - Vol. 77. - P. 026401.

23 Dharma-Wardana M.W.C., Perrot F. Equation of state and the Hugoniot of laser shock-compressed deuterium // Phys. Rev. B. - 2002. - Vol. 66. - P. 14110.

24 Dharma-Wardana M. W. C. The classical-map hyper-netted-chain (CHNC) method and associated novel density-functional techniques for warm dense matter// Int. J. Quantum Chem. - 2012. - Vol. 112. - P. 53.

25 Perrot F. and Dharma-Wardana M. W. C. Spin-polarized electron liquid at arbitrary temperatures: Exchange-correlation energies, electron-distribution functions, and the static response functions // Phys. Rev. B. - 2000. - Vol. 62. - P. 16536.

\section{References}

1 Zh.A. Moldabekov, T.S. Ramazanov, K.N. Dzhumagulova, Contrib.Plasma Phys. 52, 207-210 (2012).

2 T.S. Ramazanov, Zh.A. Moldabekov, M.T. Gabdullin, and T.N. Ismagambetova, Phys. Plasmas 21, 012706 (2014).

3 M.T. Gabdullin, T.S. Ramazanov, M.M. Muratov, T.N. Ismagambetova, G.B. Akhtanova, and J.A. Goree, Contrib. Plasma Phys. 55 (5), 366-372 (2015).

4 D.L. Goodstein States of Matter (Dover publications, Inc., 2002), 512 p.

5 S. Dutta, and J. Dufty, Phys. Rev. E 87, 032101 (2013).

6 Y. Liu, and J. Wu, The Journal of Chemical Physics 140, 084103 (2014).

7 Q. H. Du, D. Beglov, and B. Roux, J. Phys. Chem. B 104, 796 (2000).

8 P. Ballone, G. Pastore, G. Galli, and D. Gazzillo, Mol. Phys. 59, 275 (1986).

9 D. M. Duh and D. Henderson, J. Chem. Phys. 104, 6742 (1996).

10 A. Kovalenko and F. Hirata, J. Chem. Phys. 113, 2793 (2000).

11 S. Zhao, P. Feng, and J. Wu, Chem. Phys. Lett. 556, 336 (2013).

12 Y. Rosenfeld, Phys. Rev. Lett. 63, 980 (1989).

13 E. Kierlik and M. L. Rosinberg, Phys. Rev. A 44, 5025 (1991).

14 H. Lowen, Phys. Rep.-Rev. Sec. Phys. Lett. 237, 249 (1994).

15 Y.X. Yu and J.Z. Wu, J. Chem. Phys. 117, 10156 (2002).

16 S. Dutta and J. Dufty, Europhys. Lett. 102, 67005 (2013).

17 E. W. Brown, B. K. Clark, J. L. DuBois, and D. M. Ceperley, Phys. Rev. Lett. 110, 146405 (2013).

18 S. Dutta, and J. Dufty, Phys. Rev. E 87, 032102 (2013).

19 M. W. C. Dharma-Wardana, and F. Perrot, Phys. Rev. Lett. 84, 959 (2000).

20 M.W.C. Dharma-wardana, Phys. Rev. B 72, 125339 (2005).

21 M.W.C. Dharma-wardana, F. Perrot, Europhys. Lett. 63, 660 (2003).

22 M.W.C. Dharma-wardana, M.S. Murillo, Phys. Rev. E 77, 026401 (2008).

23 M.W.C. Dharma-wardana, F. Perrot, Phys. Rev. B 66, 14110 (2002).

24 M. W. C. Dharma-Wardana, Int. J. Quantum Chem. 112, 53 (2012).

25 Perrot F. and Dharma-Wardana M. W. C., Phys. Rev. B. 62, 16536 (2000). 